\title{
Biological Model
}

National Cancer Institute

\section{Source}

National Cancer Institute. Biological Model. NCI Thesaurus. Code C80519.

Systems designed to elucidate facts about complex biological phenomena, usually in a less complex or theoretical test environment. 